\title{
Jean-Jacques Rousseau : le dernier état de la Lettre à D’Alembert sur les spectacles (1758)
}

\section{Nathalie Ferrand}

\section{(2) OpenEdition}

1 Journals

\section{Édition électronique}

URL : http://journals.openedition.org/genesis/951

DOI : $10.4000 /$ genesis.951

ISSN : 2268-1590

Éditeur :

Presses universitaires de Paris Sorbonne (PUPS), Société internationale de génétique artistique littéraire et scientifique (SIGALES)

Édition imprimée

Date de publication : 10 avril 2012

Pagination : 135-157

ISBN : 978-2-84050-822-9

ISSN : 1167-5101

\section{Référence électronique}

Nathalie Ferrand, « Jean-Jacques Rousseau : le dernier état de la Lettre à D'Alembert sur les spectacles (1758) », Genesis [En ligne], 34 | 2012, mis en ligne le 10 avril 2014, consulté le 12 juin 2020. URL : http://journals.openedition.org/genesis/951 ; DOl : https://doi.org/10.4000/genesis.951 


\title{
Jean-Jacques Rousseau : le dernier état de la Lettre à D'Alembert sur les spectacles (1758)
}

\author{
Nathalie Ferrand
}

L a Lettre à D'Alembert sur les spectacles fut écrite par Rousseau dans le feu d'une polémique avec D'Alembert, contre l'idée de l'installation d'un théâtre à Genève que ce dernier venait d'avancer dans l'article « Genève » de l'Encyclopédie. Sa genèse commence donc par la lecture d'un article de l'Encyclopédie - faite par Rousseau à la fin de l'année 1757 ; elle s'achèvera à une date indéterminée, après plusieurs étapes, dans les marges d'un exemplaire de l'édition originale de 1758 (fig. 1) ${ }^{1}$ que Rousseau retouche à plusieurs reprises et dont la plupart des annotations restent inédites à sa mort, en 1778. Cet exemplaire retouché était jusqu'à présent inconnu. C'est par hasard que nous l'avons retrouvé dans les fonds anciens du Department of Special Collections de la Bodleian Library d'Oxford2.

Pourquoi se trouvait-il à Oxford ? Quelques lettres conservées $^{3}$ avec cet exemplaire éclairent en partie cette provenance et permettent de remonter au seuil du $\mathrm{XX}^{\mathrm{e}}$ siècle, lorsque ce volume appartenait à un antiquaire d'Edinburgh, William Brown. Dans une lettre datée d'avril $1902^{4}$, il se proposait de le vendre à un destinataire dont le nom n'est pas indiqué. Entré ensuite en possession de Roger Faryon Stowell (possible destinataire du précédent courrier), il est l'objet d'une demande d'expertise adressée par son épouse en 1968 à la Librairie Blackwell's d'Oxford qui, par les soins du responsable du département des livres anciens, envoie quelques photos à la Bibliothèque nationale à Paris afin de savoir si les retouches sont bien de la main de Rousseau. En juin 1968, la Bibliothèque nationale confirme l'authenticité du document et se déclare prête à acheter le volume si un prix est fixé par son propriétaire. La vente n'aura pas lieu et l'édition entrera en mai 1986 dans les collections de la Bodleian Library par donation.

L'année 1758 correspond à une période sombre de la vie de Rousseau qui vient de rompre avec ses amis
(Diderot, Grimm) et ses amours (Sophie d'Houdetot). La Lettre à D'Alembert consomme une rupture avec le camp des philosophes et donne forme au mythe, si durable pour Rousseau, de Genève comme lieu encore intact à préserver des influences néfastes du théâtre et de l'artifice. Cette lettre ouverte à D'Alembert - mais qui en réalité a des destinataires multiples : Voltaire que Rousseau soupçonne d'être à l'origine de l'article, Diderot dont Rousseau ne partage pas les idées sur le théâtre et qu'il accuse dans une note de la préface d'avoir trahi leur amitié en révélant des secrets -, Rousseau la présente comme un écrit d'outre-tombe, jeté sur le papier par un écrivain qui peine à se survivre à lui-même : "Lecteur, si vous recevez ce dernier ouvrage avec indulgence, vous accueillirez mon ombre ; car pour moi, je ne suis plus 5 . » Et pourtant, la vivacité dans l'écriture est intacte et les effets littéraires sont

1. J.-J. Rousseau citoyen de Genève, À Mr. D'Alembert, De l'Académie Françoise, de l'Académie Royale des Sciences de Paris, de celle de Prusse, de la Société Royale de Londres, de l'Académie Royale des Belles-Lettres de Suede, \& de l'Institut de Bologne : Sur son Article Genève Dans le VIIme. Volume de l'Encyclopédie, Et particulièrement, Sur le projet d'établir un théâtre de comédie en cette Ville. Dii meliora piis, erroremque hostibus illum., À Amsterdam, Chez Marc Michel Rey, M.DCC.LVIII, $264 \mathrm{p}$.

2. Sous la cote Arch. H e.146.

3. Sous la cote Arch. H e.146*.

4. "Dear Sir, I have the word of an expert and I have myself made some comparison with a letter of Rousseaus', and you may rest assured that it is as I describe it. I bought it privately from one who has many ASS [lettres autographes] passing through his hands. Of course you can refer to specimens in the British Museum. As you will see, it is not the kind of things forgers would make, and the writing is characteristic. Yours faithfully, D. Mackintosh W. Brown. »

5. Jean-Jacques Rousseau, Lettre à D'Alembert sur les spectacles, dans Euvres complètes, t. V, Paris, Gallimard, coll. «Bibliothèque de la Pléiade », 1995, Préface, p. 7. L'orthographe « accueillirez » est souhaitée par Rousseau, malgré la correction que veut apporter l'éditeur. 
très calculés. Rousseau, vivant bien plus longtemps que ne l'annonçait le pathos de sa préface, continuera à remanier pendant plusieurs années, comme on va le voir, ce texte au-delà de sa première publication et des circonstances particulières de sa première rédaction. Un manuscrit de travail autographe (une mise au net retouchée) de la Lettre à D'Alembert a été conservé, il est en mains privées, et avait pu être consulté par Bernard Gagnebin et Jean Rousset pour leur édition de ce texte au tome $\mathrm{V}$ des Euvres complètes de JeanJacques Rousseau publiées chez Gallimard (Pléiade) en $1995^{6}$; deux feuillets séparés tirés d'une autre mise au net autographe avaient également été retrouvés par Ralph Leigh à la Bibliothèque nationale 7 . En revanche, le dernier état retouché de la Lettre n'existait jusqu'à maintenant qu'à titre d'hypothèse. Notant des variations importantes entre l'édition de 1758 et celle qui fut donnée de manière posthume en 1781 dans la Collection complète des Euvres de J.-J. Rousseau (tome VI de l'édition in-quarto), les éditeurs de la Pléiade supposaient : «Les éditeurs Moultou et Du Peyrou ont dû transcrire [des notes et corrections] d'un exemplaire annoté par Rousseau, qu'ils avaient en main ${ }^{8}$. »

Non seulement cet exemplaire annoté a bel et bien existé, mais en plus il a survécu jusqu'à aujourd'hui. Accessible aux chercheurs, il permet désormais de prendre la mesure de ce qui s'est joué à ce stade de la révision de la Lettre. Son intérêt est multiple, d'abord du point de vue de l'établissement du texte, puisqu'il présente le dernier état revu et corrigé par l'auteur et offre donc aux éditeurs une version de l'œuvre qui doit servir de base pour la publication du texte final souhaité par Rousseau. Mais surtout dans la perspective génétique qui nous occupe, cet exemplaire retouché nous met face à un document de travail très spécifique où Rousseau, de nouveau la plume à la main, remanie son texte. Il nous offre un exemple de la manière dont l'imprimé se transforme chez Rousseau, comme chez d'autres auteurs, en espace de travail génétique, relançant la dynamique de l'écriture ; il nous permet de regarder de près le statut des retouches et la forme de dialogisme que Rousseau institue avec son œuvre quand il s'annote lui-même et revient sur un état achevé d'un de ses textes. De quelle nature est ce remaniement?
Peut-on y détecter plusieurs moments intellectuels ? Dans la mesure où la rédaction de la Lettre à D'Alembert fut liée à l'actualité bien précise d'une querelle littéraire, quel sens prennent des ajouts faits après la bataille : visent-ils à rester dans la polémique ou bien à la dépasser ?

\section{De l'errata de 1758 à la fabrique des œuvres complètes de 1781}

La Lettre à D'Alembert est dès sa publication un texte marqué par les repentirs. Alors que le volume est sous presse au cours de l'été 1758, Marc-Michel Rey reçoit de Rousseau tant de corrections et d'additions qu'il est contraint de publier son édition accompagnée d'un «Avis de l'imprimeur » et d'un errata qui en donnent le détail. Il explique :

Mr. Rousseau m'ayant adressé les corrections \& les additions suivantes pour être placées en leur lieu, je n'ai pu les faire y entrer, ces feuilles étant déja toutes imprimées. Je crois faire plaisir au public \& remplir les vues de l'Auteur en les ajoutant à la fin de son ouvrage. À Amsterdam le 15. Juillet 1758 .

C'est que la Lettre à D'Alembert est un texte particulièrement travaillé dans le détail de l'écriture. Elle correspond au moment où Rousseau formule ses idées sur l'importance de l'harmonie en matière de style. Le 8 juillet, corrigeant les épreuves, il écrit à son éditeur :

page 186, ligne 5 en remontant femmes je n'avois point mis cette $s$, otez-la ; vous me direz qu'elle est fort indifférente, et vous avez raison quant au sens ; mais outre que le singulier est plus élegant, ce pluriel ajoûte dans la phrase une sillabe qui en gâte absolument l'harmonie, et l'harmonie

6. Rousseau, Lettre à D'Alembert, dans CEuvres complètes, éd. citée, p. 1811-1812.

7. Ralph Leigh, «Les manuscrits disparus de J.-J. Rousseau. Quelques observations et quelques fragments retrouvés ou complétés », dans Annales J.-J. Rousseau, $\mathrm{n}^{\circ}$ 34, 1956-1958, p. 31-81. Voir notamment p. 77-81.

8. Rousseau, Lettre à D'Alembert, dans CEuvres complètes, éd. citée, p. 1814 . 
me paroit d'une si grande importance en fait de stile que je la mets immediatement après la clarté, même avant la correction $^{9}$.

Mais une fois le livre paru, cet interminable processus de révision ne cesse pas : Rousseau continue à communiquer de nouvelles modifications à Rey en vue d'autres éditions 10 et surtout, il annote son exemplaire personnel.

Tel qu'il nous est parvenu, l'exemplaire corrigé par Rousseau de la Lettre à D'Alembert porte une centaine de retouches (corrections orthographiques, ajouts, substitutions, suppressions) ${ }^{11}$. Mais toutes les annotations ne sont pas de Rousseau lui-même et il importe donc d'abord de les distinguer soigneusement les unes des autres pour concentrer l'analyse sur ce qui relève de sa révision personnelle. Trois mains différentes sont identifiables en plus de celle de l'auteur - lequel, pour compliquer un peu plus les choses, utilise lui-même une gamme variée d'écritures qu'on peut heureusement identifier assez nettement grâce à des manuscrits autographes datant de la même période, ceux de La Nouvelle Hélö̈se (rédigée entre 1756 et 1760)12. L'apparition des retouches allographes est liée au fait que l'exemplaire personnel est passé à un certain moment entre les mains des responsables de la Collection complète des Euvres de J.-J. Rousseau, édition qui allait être publiée de manière posthume (Rousseau étant mort en 1778) à partir de 1780 à Genève. À quel moment est-il sorti des mains de Rousseau ? Peut-être en 1765, lors de la fuite de Môtiers, lorsque Rousseau remit à son ami Du Peyrou «quelques paquets de livres, de lettres et de manuscrits », manuscrits qui selon Raymond Birn «incluaient des révisions d'ouvrages publiés 13 ». L'exemplaire personnel porte bien les traces concrètes du travail de préparation de cette édition : on y trouve inscrits en marge des renvois à d'autres volumes de la Collection $^{14}$ (p. 1, fig. 2), ou sur une autre page un échange qui dit l'embarras des éditeurs face à une note de Rousseau (p. 142, fig. 3). Ont également été reportés à la main avec une calligraphie remarquable, dans les marges du texte, les changements qui figuraient dans l'Avis de juillet 1758 imprimé à la fin du livre.
Les pages de l'Avis et de l'errata ont été biffées. Cette incorporation au fil du texte de la liste d'additions et de corrections, qui sont alors placées au bon endroit, allait rendre plus aisée la composition de l'édition par les ouvriers d'imprimerie. Guidés par des principes de cohérence, de lisibilité et de complétude, les éditeurs de 1781 ont également inséré un ajout que Rousseau n'avait pas noté lui-même mais qui apparaissait dans une édition antérieure (Rey, 1762, p. 73, fig. 4). De la sorte ils ont réalisé un exemplaire complet, qui a ensuite été suivi pour l'impression, à une omission près (p. 57, fig. 5) 15 .

Par conséquent, s'il nous introduit dans l'atelier de l'écrivain au travail, ce volume annoté nous ouvre aussi à une autre dimension, celle de l'atelier typographique et de la fabrication concrète de la Collection complète des Euvres de J.-J. Rousseau par la Société typographique de Genève. Deux dynamiques sont donc à l'œuvre dans les marges de cet exemplaire, qu'il ne faut pas confondre

9. Correspondance complète de Jean-Jacques Rousseau, édition critique établie et annotée par R. A. Leigh (dorénavant $C C$ ), 52 vol., Genève, Madison, Banbury, Oxford, Voltaire Foundation, 1965-1998, lettre n ${ }^{\circ} 667$. Le passage en question se trouve à Rousseau, Lettre à D'Alembert, dans Euvres complètes, t. V, éd. citée, p. 91.

10. Voir par exemple les lettres à Rey du 14 mars 1759 et du 6 mars 1760. 11. Si on le confronte à d'autres exemplaires d'œuvres imprimées que Rousseau a annotés en marge (le Discours sur l'origine de l'inégalité, le Contrat social et La Nouvelle Héloïse), c'est le volume le plus richement révisé.

12. N. Ferrand, «Dans 1'atelier de La Nouvelle Hélö̈se. Rassembler, transcrire, interpréter les manuscrits de J.-J. Rousseau », dans Le Modèle de Julie. Sources et postérités de "La Nouvelle Héloüse », dir. Geneviève Goubier et Stéphane Lojkine, Paris, Desjonquères, à paraître, 2012.

13. Raymond Birn, «Les "CEuvres complètes" de Rousseau sous l'Ancien Régime », Annales Jean-Jacques Rousseau, n 41, 1997, p. 231-264, citations aux pages 238 et 239.

14. « L'article GENÈVE qui a donné lieu à cette lettre de M. Rousseau, sera imprimé dans le premier volume du Supplement, avec les autres pièces qui y ont rapport. »

15. Une substitution de Rousseau, qui remplace à la page 57 (p. 35 dans l'édition de la Pléiade) « tout le monde » par «tout le genre humain », n'a pas été suivie par les éditeurs de 1781. Il s'agit sans doute d'un oubli, à moins que ce soit une réticence face à une formule désormais presque caricaturale du lexique de Rousseau. Voltaire se moquait de Rousseau disant « le genre humain et moi ». 
même si elles cohabitent de manière intéressante et suggestive : la réflexion d'un auteur qui revient sur l'un de ses textes et qui relève d'une approche génétique de l'œuvre ; et le travail des éditeurs préparant une version du texte qu'ils s'apprêtent à publier et qui relève de l'histoire du livre et de l'édition ${ }^{16}$. Un horizon et une intentionnalité leur sont communs : les interventions allographes visent à préparer une prochaine édition ; Rousseau à ce stade s'annote et se corrige non pas dans un tête-à-tête intime et solitaire avec son œuvre mais bien parce qu'il pense lui aussi à une nouvelle publication et donc à un regard externe sur ses retouches ${ }^{17}$.

\section{Les autocorrections de Rousseau : minuties et enjeux de sens}

Les interventions de Rousseau sur son exemplaire personnel de l'édition originale de la Lettre à D'Alembert portent sur différents niveaux : elles vont de la banale correction d'une coquille à l'insertion de notes en bas de page qui vont enrichir le sens par une référence à des textes jusqu'alors non mentionnés ou de notes qui vont rectifier une position que Rousseau finit par désavouer. L'histoire de cette révision peut en partie être connue par des croisements avec la correspondance jusqu'en 1764. Il est frappant de constater combien Rousseau reste en rapport prolongé avec ce texte déjà publié, comme si la publication ne rompait en rien le lien génétique de l'auteur avec sa création et qu'elle restait en permanence présente à son esprit. De plus l'impression ne fige pas l'auteur dans une position définitive par rapport à ce qu'il a pensé et rendu public, mais le laisse libre de se corriger à l'intérieur des pages déjà écrites et imprimées. Il y revient, en fonction d'événements extérieurs (une lettre qu'on lui écrit, un livre qu'il lit...) ou selon l'évolution de sa pensée, et il se donne même, comme on le verra, le droit de se démentir lui-même de manière spectaculaire.

Pour Rousseau, un $s$ de plus ou de moins engage l'harmonie et donc l'effet d'une phrase. Aussi n'estil pas surprenant qu'en de nombreux endroits de son exemplaire, il retouche l'orthographe ou le rythme d'une phrase, change l'ordre des mots, déplace une virgule ou mette un point ${ }^{18}$. Il corrige des fautes mais modifie aussi de manière systématique la graphie de certains mots, comme le mot «mistère » ou l'adjectif « mistérieuse » qui se voient pourvus systématiquement d'un $y$ à la place du $i$ - graphie qui apparaît dans sa correspondance personnelle à partir de 1761, alors qu'auparavant il utilise l'autre. En mars 1760, il s'avise, avec un certain retard, d'une erreur de français pourtant très visible puisqu'elle apparaissait sur la page de titre et au titre courant. Il fait alors la leçon à son éditeur :

Je dois vous avertir aussi, pendant que j'y pense, que quand le mot de Monsieur s'écrit en abrégé, il n'y faut point d'r. Mr mais seulement une $\mathrm{M}$ et un point. M. Je vous avertis de cela parce que dans ma Lettre à $\mathrm{M}$. D'Alembert on a constamment mis dans l'imprimé cette $r$ qui n'étoit point dans la copie ${ }^{19}$.

16. De ce point de vue ajoutons encore que l'exemplaire personnel de Rousseau de la Lettre à D'Alembert conservé à la Bodleian Library d'Oxford appartient à un recueil composite : sous une reliure faite tardivement, il est suivi d'une édition sans date, lieu ou nom d'éditeur, d'un autre texte sur le théâtre, publié sous le nom de Rousseau en 1763, De l'imitation théâtrale, qui allait être réédité dans le même tome de la Collection complète des CEuvres de J.-J. Rousseau à la suite de la Lettre à D'Alembert. Ce texte imprimé porte lui aussi des traces dans ses marges, mais d'une tout autre nature, puisqu'il s'agit de signatures au crayon correspondant probablement aux noms des ouvriers typographes qui s'occupèrent de la composition matérielle de ce texte. Parmi les noms apparaissent ceux de : Lambert, Binot, Frolin, Laurens, Livron. La reliure est tardive et les pages ont été maladroitement rognées, supprimant la fin de ligne de quelques notes marginales.

17. Rousseau prévoyait de réaliser lui-même une édition générale de son œuvre et à cette fin il préparait soigneusement des exemplaires révisés et corrigés de ses ouvrages. Cette édition devait lui assurer un revenu, ainsi qu'à Thérèse après sa mort. Voir à ce sujet l'étude éclairante de R. Birn déjà citée. 18. Parmi les nombreuses corrections ou changements orthographiques : « recommendation » devient « recommandation »; « assés » devient systématiquement «assez »; « essentiel » est corrigé en « essenciel»; « ches » devient « chez »; « tirans », «tyrans »; « tranquiles », « tranquilles »; « spatieuses » devient « spacieuses »; « paie », « paye »; « poliçons » devient « polissons »; « poliçonneries », « polissoneries »; « gaieté » devient « gaité », etc. Parmi les changements stylistiques, « Il n'y a point d'art pour faire naître cet intérêt » par « Il n'y a point d'art pour produire cet intérêt » (p. 28) ; «qu'une scene si touchante soit animée des sentimens tendres \& pathétiques que le sujet fournit » devient « qu'une scene si touchante soit animée des sentimens tendres \& pathétiques que fournit la matière » (p. 91) ; «comme les dés qui partent de la main, quelque impulsion qu'on leur donne, n'en amenent pas plus aisément le point qu'on desire » devient « comme les dés qui partent de la main, quelque impulsion qu'on leur donne, n'en amenent pas plus aisément le point désiré » (p. 133), etc.

19. Lettre à Rey, 6 mars 1760, CC, lettre n ${ }^{\circ} 952$. 
Dans son exemplaire, il a biffé le $r$ sur la page de titre et sur les titres courants. Si l'auteur suisse en remontre à son éditeur d'Amsterdam au sujet de l'abréviation de Monsieur en français, c'est que la pureté de son français, conquise au prix d'efforts et d'une certaine crispation linguistique qu'il saura retourner à son avantage dans La Nouvelle Héloïse en ayant délibérément recours aux provincialismes de ses épistoliers, est un enjeu qui regarde son positionnement littéraire, intellectuel et sociologique 20 .

En mars 1759, apprenant que Rey est sur le point de republier son livre à son insu, il lui écrit sèchement et en profite pour réclamer la substitution d'un passage de la Lettre:

j'ai un changement à faire qui n'est pas considérable, mais qui m'importe. Je vous prie de vouloir bien faire un carton 21 même deux s'il le faut, pour cela, et avoir soin que ce carton soit à tous les exemplaires que vous enverrez en France. Que si vôtre envoi étoit déja fait, je souhaite ni plus ni moins que le carton soit fait et mis à deux ou trois exemplaires que je vous prie de m'envoyer ainsi corrigés. C'est une affaire qui m'intéresse personnellement. Voici le changement. page 73. de la pre Edition ligne 8 . après ces mots femmes sans mours. ôtez les huit lignes suivantes et substituez leur ce qui suit : Ce sont eux qui les prémiers ont introduit ces grossiéres équivoques, non moins proscrittes par le goût que par l'honnêteté, qui firent longtems l'amusement des mauvaises compagnies, l'embarras des personnes modestes, et dont un meilleur ton, lent dans ses progrès, n'a pas encore purifié certaines provinces. D’autres Auteurs, plus réservés dans leurs saillies, laissant les prémiers amuser les femmes perdües, se chargérent d'encourager les filoux. Regnard un des moins libres n'est pas le moins dangereux. C'est chose incroyable \&c. 22 .

Rey tiendra compte de cette demande de Rousseau dans l'édition de 1762 . De quoi s'agit-il précisément? Si l'on se reporte à la page 73 de son exemplaire personnel, on aperçoit le passage retravaillé (fig. 4). Il apparaît dans un développement sur la comédie dont Rousseau fustige l'immoralité. S'en prenant à Molière, dont il reconnaît néanmoins le génie, il dresse un portrait à charge de deux de ses successeurs à la fin du XVII ${ }^{e}$ siècle : Dancourt, qui s'est illustré dans la farce et Regnard dans la haute comédie. Sur son exemplaire, Rousseau a biffé le passage suivant :
Je ne ferai pas à Dancourt l'honneur de parler de lui : ses Pieces n'effarouchent pas par des termes obscenes, mais il faut n'avoir de chaste que les oreilles, pour les pouvoir supporter. Regnard, plus modeste, n'est pas moins dangereux : laissant l'autre amuser les femmes perdues, il se charge, lui, d'encourager les filoux.

$\mathrm{Au}$ bas de page, Rousseau a introduit une variante, qui n'est pas exactement celle de la lettre de mars 1759. C'est une version intermédiaire (inconnue) qui vient se glisser à la fin de la phrase précédente :

[les successeurs de Molière qui] substituant au vrai talent l'esprit du libertinage se parèrent de ces mots à double entente, et de ces grossières équivoques non moins proscrittes par le goût que par l'honnêteté qui firent longtems l'amusement des mauvaises compagnies, et dont le meilleur ton, lent dans ses progrès n'a pas encore purifié certaines provinces.

Latéralement on trouve la version qui correspond au contenu de la lettre de Rousseau à Rey de mars 1759 et à celle de l'édition de 1762 - elle a été reportée par une autre main que celle de Rousseau. En fin de compte, le nom de Regnard a été réintroduit mais pas celui de Dancourt qui, et c'est le principal changement survenu au cours de cette succession de retouches, se trouve effacé du livre. Pour justifier auprès de son éditeur ce changement «qui [lui] importe », Rousseau invoque sans les préciser des raisons personnelles («C'est une affaire qui m'intéresse personnellement »). Quelles peuvent-elles être ? Dans son premier texte, Rousseau faisait allusion à Florent Carton, dit Dancourt (16611725), comédien et dramaturge, auteur notamment du Chevalier à la mode qui met en scène un escroc dupant de riches veuves, dont les pièces sont encore très jouées au temps de Rousseau. Mais en 1759, un autre Dancourt est entré sur la scène littéraire internationale : Louis Heurteaux, dit Dancourt (1725-1801), acteur

20. Voir les analyses de Jérôme Meizoz, Le Gueux philosophe (JeanJacques Rousseau), Lausanne, Antipodes, 2003, p. 35 sq.

21. «Feuillet imprimé après coup, inséré dans un livre en lieu et place d'un autre et offrant un texte modifié », Christian Galantaris, Manuel de bibliophilie, Paris, Éditions des Cendres, 1997, p. 49.

22. Lettre à Rey, 14 mars $1759, C C$, lettre n ${ }^{\circ} 788$. 
et dramaturge lui aussi. Homonyme du précédent, L. H. Dancourt vient de publier à Berlin une lettre ouverte à Rousseau intitulée L.-H. Dancourt Arlequin de Berlin à Mr. J. J. Rousseau, citoyen de Genève : c'est une réplique mordante à la Lettre à D'Alembert depuis le point de vue d'un comédien, d'un homme qui a une connaissance approfondie du répertoire, l'expérience de la scène et celle des réactions du public. Cet ouvrage de plus de trois cents pages, qui prend le temps de citer point par point Rousseau mais aussi de nombreux textes de théâtre à l'appui de sa démonstration, est une réfutation en règle de la Lettre à D'Alembert. On peut comprendre que Rousseau n'ait pas souhaité que ses lecteurs puissent confondre les deux Dancourt et qu'à ce stade, ce nom soit devenu véritablement indésirable.

Une autre suppression significative dans le texte apparaît dans un passage sous haute tension puisqu'il s'agit de celui où Rousseau répond indirectement à la formule célèbre de Diderot qui l'avait blessée dans Le Fils naturel en 1757, « Il n'y a que le méchant qui soit seul » (p. 55, fig. 6). Dans la page où Rousseau explore le profil du méchant à travers celui du misanthrope, un véritable « monstre » qui ferait horreur s’il existait, dont la haine est « une dépravation de la nature \& le plus grand de tous les vices », il fait disparaître de son propos l'explication suivante : «puisque, toutes les vertus sociales se rapportant à la bienfaisance, rien ne leur est si directement contraire que l'inhumanité ». Cette suppression 23 correspond à une évolution de la pensée de Rousseau au sujet de la bienfaisance, qui au fil des années perd son caractère central et positif. Rousseau jette un regard lucide sur les contraintes qu'elle comporte et l'obligation de reconnaissance qu'elle engendre - ainsi qu'il l'éprouve lors du séjour chez Mme d'Épinay et qu'il l'écrit à Malesherbes en 1762 : « car tout bienfait exige reconnoissance ; et je me Sens le cœur ingrat par cela Seul que la reconnoissance est un devoir ${ }^{24}$ ». Peu à peu il ne la considère plus comme une vertu - «Contenter le goût qui nous porte à bien faire est bonté, mais non pas vertu » explique-t-il plus tard au jeune Franquières 25 . Comme l'a montré Jean Starobinski, le caractère ostentatoire et éventuellement feint - songeons aux Liaisons dangereuses de Laclos plus tard et à l'épisode de fausse bienfaisance de Valmont (lettre XXI) - qu'elle revêt au XvIII e siècle finit par susciter sa méfiance 26 . Cette suppression est la marque d'un assombrissement de sa pensée concernant les valeurs qui tissent la vie sociale.

\section{Être à soi-même son propre Aristarque. Le rôle des notes en bas de page}

Dans la Lettre à D'Alembert, Rousseau fait pour la première fois l'expérience d'une voix singulière 27 , la sienne propre. Pour la première fois, il publie un texte sans l'avoir soumis au regard extérieur de celui qui fut longtemps son lecteur et son plus avisé critique, Diderot. Sa préface est transparente pour les lecteurs de l'époque : «Vivant seul, je n'ai pu le montrer à personne. J'avois un Aristarque 28 sévére et judicieux, je ne 1'ai plus, je n'en veux plus ; mais je le regreterai sans cesse, et il manque bien plus encore à mon cœur qu'à mes Écrits ${ }^{29}$. » Suit une note assassine en latin par laquelle il prend

23. Ce passage se trouve à la page 34 dans l'édition de la Pléiade. La suppression, dont l'édition de 1781 a bien tenu compte, est passée inaperçue aux éditeurs de la Pléiade.

24. Lettre à Malesherbes du 4 janvier 1762, $C C$, lettre $n^{\circ} 1622$.

25. Lettre à Laurent Aymon de Franquières, 15 janvier 1769, $C C$, lettre $\mathrm{n}^{\circ} 6529$.

26. Jean Starobinski, Largesse, Paris, Réunion des musées nationaux, 1994.

27. Au livre X des Confessions, Rousseau insiste sur « le ton singulier qui régne dans cet ouvrage, et qui tranche si prodigieusement avec celui du précédent [le Discours sur l'origine de l'inégalité] », voir Les Confessions, dans Euvres complètes, Paris, Gallimard, coll. «Bibliothèque de la Pléiade », 1959, t. I, p. 496.

28. « ARISTARQUe, s. m. (Hist. \& Littérat.) dans sa signification littérale, signifie un bon prince [...] mais on le prend ordinairement pour un critique éclairé \& severe, parce qu'un grammairien nommé Aristarque, fit une critique solide \& sensée des meilleurs poëtes, sans en excepter Homere. Un Aristarque signifie donc un censeur; \& cette expression étoit déjà passée en proverbe du tems d'Horace » (Encyclopédie).

29. Lettre à D'Alembert, dans Euvres complètes, éd. citée, Préface, p. 7. Sur la présence de la main de Diderot dans les premiers écrits de Rousseau, voir une confidence tardive de Rousseau : «Diderot, dont j'étudiois particuliérement la diction quand je commençai d'écrire, et qui même a mis dans mes premiers ouvrages plusieurs morceaux qui ne tranchent point avec le reste, et qu'on n'en Sauroit distinguer du moins quant au style » (lettre à Claude Aglancier de Saint-Germain, 26 février 1770, CC, lettre $n^{\circ} 6674$, nous soulignons). 
congé de Diderot. Expérience d'écriture libératoire par laquelle Rousseau gagne une autonomie littéraire, la Lettre à D'Alembert a constitué aussi, dans le même temps, un deuil douloureux de sa gémellité intellectuelle et scripturale avec Diderot.

Seul devant son texte, on comprend qu'il redouble d'efforts pour le parfaire, avant et même après la première publication. Mais cette voix singulière conquise par le choc d'une rupture et dans la solitude, se dédouble et laisse percer, au fil des retouches, des voix d'abord absentes. En effet, parmi les retouches les plus significatives qui apparaissent au fil du temps dans l'exemplaire personnel, se trouve une série de cinq nouvelles notes en bas de page qui vont faire intervenir des textes et des auteurs jusqu'alors non mentionnés, et ainsi infléchir la position de l'auteur face à son propre discours. C'est sur ce niveau de parole très particulier offert par l'annotation infrapaginale, et sur son évolution, que se concentrera la dernière partie de nos analyses.

En matière de notes en bas de page, Rousseau n'a rien d'un novice, comme le montre le dispositif argumentatif complexe du Discours sur l'origine de l'inégalité30. Il est plutôt dans ce domaine un virtuose qui mérite une mention particulière dans une histoire de la note de bas de page telle qu'Anthony Grafton en avait posé les bases ${ }^{31}$. La Lettre à D'Alembert est dès le départ un texte lourdement enrichi de notes qui viennent étayer le propos de l'auteur. « Le texte persuade, la note prouve », affirme A. Grafton en observant les pratiques érudites et celles des philosophes de la première modernité, et l'on peut dire que dans sa première version, le texte de Rousseau vérifie grosso modo cet axiome. Les choses changent dans la révision ultérieure à l'œuvre sur l'exemplaire personnel. En le modifiant, Rousseau touche à l'équilibre argumentatif délicat qui unit le corps de son texte et ses notes, il le perturbe, voire le subvertit d'une manière inattendue.

Sur l'exemplaire personnel, on peut distinguer plusieurs familles de retouches infrapaginales qui semblent relever d'une même campagne d'écriture : même encre, même ductus, même positionnement. Certaines constituent des notes entièrement nouvelles (p. 92, 95, 141, fig. 7, 8, 9) ; d'autres viennent prolonger certaines notes déjà existantes (p. 176, 188, 201, fig. 10, 11 et 12).
Examinons le premier ensemble. Ces trois notes des pages 92, 95 et 141 semblent avoir été posées sur la page dans un même moment - ce qui ne veut pas dire qu'elles ont été conçues au même moment. Elles ont aussi en commun d'avoir la même fonction : enrichir les références littéraires de la Lettre à D’Alembert. En effet, ces notes mentionnent chacune des textes qui jusqu'alors n'avaient pas été cités : dans l'ordre, Pamela de Richardson, Le Marchand de Londres de Lillo et Iphigénie en Aulide d'Euripide.

« Il y a, dans le 7e tome de Pamela, un examen très judicieux de l'Andromaque de Racine, par lequel on voit que cette piece ne va pas mieux à son but prétendu que toutes les autres », écrit Rousseau au bas de la page 9232 de son exemplaire (fig. 7). Il fait allusion à un très long passage du célèbre roman de Richardson 33 où Pamela désormais mariée et vivant dans la haute société raconte dans une lettre l'effet sur elle d'Andromaque qu'elle vient de voir au théâtre à Londres ${ }^{34}$. C'est au début de l'année 1762 que Rousseau a, selon toute vraisemblance, lu Pamela ainsi que nous l'apprend la correspondance 35 .

30. Rappelons que le Discours sur l'origine de l'inégalité (1755) s'illustre par un dédoublement de la parole en deux blocs : le texte principal, puis un corps de Notes explicatives destinées à ceux qui veulent approfondir leur lecture, chacun (texte et Notes) étant pourvu à son tour... de notes en bas de page.

31. Anthony Grafton, Les Origines tragiques de l'érudition. Une histoire de la note en bas de page, Paris, Éditions du Seuil, 1998 [The footnote: a curious history, 1997]. Rousseau n'est pas mentionné dans cette étude. Signalons que dans son ouvrage Du livre au lire. "La Nouvelle Héloïse » roman des Lumières, Paris, H. Champion, 2002, Yannick Séité a consacré des analyses pionnières aux notes de La Nouvelle Héloïse, voir p. 273-350. Il montre à quel point les notes sont l'objet d'une attention particulière de Rousseau (p. 283-284). Dans Séditions infrapaginales. Poétique historique de l'annotation littéraire (XVII -XXIe siècles) (Genève, Droz, 2011) Andréas Pfersmann a situé les notes de Rousseau à La Nouvelle Héloïse dans l'histoire longue de cette pratique littéraire, voir p. 203-212.

32. Ce passage correspond à la page 50 dans l'édition de la Pléiade.

33. Pamela or virtue rewarded, parut en 1740, et fut suivi de Pamela in her exalted condition en 1742. L'épisode évoqué appartient à la seconde partie des aventures de Pamela.

34. Pamela, ou la vertu récompensée, traduit de l'anglais, tome septième, Amsterdam, Aux dépens de la Compagnie, 1743, lettre XI, p. 70-94. Il s'agit d'un très long passage qui passe au crible cette pièce et les effets moraux qu'elle peut avoir sur une spectatrice, pour en souligner les méfaits. 
Dès 1758, dans l'édition originale de la Lettre à D'Alembert, Rousseau mentionnait déjà un autre roman de Richardson, Clarisse Harlowe, dans une note très admirative, même s'il allait prendre ses distances avec ce roman quelque temps plus tard, dans La Nouvelle Héloïse (1761). Mais mentionner Richardson en 1758 est une chose, le mentionner après 1762, c'est tout différent. Entre ces deux dates, Diderot a publié un fracassant Éloge de Richardson dans le Journal étranger et a mis l'auteur anglais en lumière pour mieux jeter l'obscurité sur le tour de force romanesque que vient d'accomplir son ancien ami Rousseau avec La Nouvelle Héloïse. Aussi mentionner positivement Pamela, ce n'est pas seulement rendre un hommage à Richardson, c'est faire entendre inévitablement aussi la voix de Diderot. Dans l'œuvre qui a scellé leur rupture, Rousseau esquisse ainsi une forme de dialogue avec Diderot, certes ambigu ${ }^{36}$, mais dialogue tout de même.

Même retour, moins ambigu cette fois, à Diderot à travers la mention enthousiaste du Marchand de Londres (1731) de Lillo qui se trouve insérée à la page 95 de l'exemplaire personnel (fig. 8) ${ }^{37}$. Rousseau ne ménage pas son éloge : «Ajoutons le Marchand de Londres, piéce admirable et dont la morale va plus directement au but qu'aucune piéce françoise que je connoisse. » Là encore, derrière la référence anglaise, se cache une référence à Diderot qui dans le premier de ses Entretiens sur le Fils naturel de 1757, voyait dans cette pièce un modèle du drame bourgeois, cette forme théâtrale nouvelle et intermédiaire que Diderot appelle de ses vœux pour moraliser le théâtre. Or l'idée de moraliser le théâtre était l'objet d'un désaccord avec Rousseau ${ }^{38}$. Dans la Lettre à D'Alembert, il prenait le contre-pied de Diderot et récusait l'utilité de nouvelles formes théâtrales moralisantes, des pièces qui « instruisent beaucoup, si l'on veut ; mais elles ennuient encore davantage. Autant vaudroit aller au sermon 39 ». Aussi cette addition marginale marque-t-elle un revirement et suggère que Rousseau a tempéré au fil du temps son hostilité aux propositions de Diderot sur la rénovation du théâtre.

La troisième référence littéraire que Rousseau ajoute en bas de page un peu plus loin dans son exemplaire (p. 141, fig. 9), n'est ni contemporaine ni anglaise, mais renvoie à sa culture classique. Elle s'agrège à un long passage sur la profession de comédien, dont Rousseau cherche à montrer qu'elle est déshonorante pour qui l'exerce et que Genève, pour cette raison, ne peut admettre l'arrivée d'une troupe de comédiens qui mettrait en danger la moralité de ses citoyens. Certes autrefois la Grèce antique reconnaissait aux acteurs une dignité qui leur permettait d'occuper certaines charges publiques, dans l'État ou dans les Ambassades. Mais elle fait figure d'exception. Pour mieux isoler historiquement cette exception qui pourrait nuire à sa démonstration, Rousseau énumère six raisons qui l'expliquent dans le contexte très précis de cette culture. Si les acteurs étaient honorés, c'est que la tragédie grecque tirait ses sujets de l'histoire nationale et instruisait le peuple par de grands tableaux presque sacrés. Au point 4 de son énumération, il glisse un astérisque : « $4^{\circ}$. Ce Peuple, enthousiaste de sa liberté jusqu'à croire que les Grecs étoient les seuls hommes libres par nature, ${ }^{*}$ se rappelloit avec un vif sentiment de plaisir ses anciens malheurs \& les crimes de ses Maîtres. » En bas de la page : "* Iphigénie le dit en termes exprès dans la Tragedie d'Euripide qui porte le nom de cette Princesse ${ }^{40}$. » Rousseau se réfère à un passage très précis d'Iphigénie en Aulide, qu'il n'a pas besoin de citer tant ce texte devait être familier à ses lecteurs. Iphigénie sur le point d'être sacrifiée s'exclame avec un courage viril qui devait certainement faire vibrer l'âme de Rousseau : "Je me dévoue. Grecs, me voici prête, sacrifiez-moi, \& renversez Troie ! Vos trophées feront ma gloire, \& me tiendront lieu pour toujours d'hymen, d'époux \& de postérité. L'ordre veut

35. Ce livre lui est envoyé par Pierre Guy de La Roche le 6 février 1762, de la part de la Maréchale de Luxembourg, voir CC, lettre n 1667.

36. Soulignons en effet le caractère ambigu, voire retors de cette note : si Richardson est bien ce grand génie que proclame Diderot, si ses romans sont exemplaires et disent la vérité, alors ce qu'il dit dans Pamela des effets négatifs du théâtre à travers l'exemple de l'Andromaque de Racine est juste. Par conséquent, moi Rousseau qui critique le théâtre et les méfaits de la tragédie, j'ai raison.

37. Ce passage correspond à la page 52 dans l'édition de la Pléiade. 38. Voir à ce sujet Nicholas Cronk, « Dorval et le dialogue à trois voix : la présence de Rousseau et de Voltaire », dans Études sur "Le Fils naturel » et les «Entretiens sur le Fils naturel » de Diderot, dir. N. Cronk, Oxford, VIF, 2000, p. 126 et 135.

39. Lettre à D'Alembert, dans Euvres complètes, éd. citée, p. 43. 
que les Grecs commandent aux barbares, \& non les barbares aux Grecs. Ceux-là sont nés pour l'esclavage ; \& ceux-ci pour la liberté41. »

Les additions manuscrites de Rousseau à la Lettre à D'Alembert introduisent donc la voix d'auteurs avec lesquels Rousseau noue une forme de dialogue différé, Richardson au sujet de Racine, Lillo au sujet du drame bourgeois, Euripide sur l'enthousiasme de la liberté, et Diderot de manière indirecte. Mais aussi, et c'est plus troublant, on entend la voix de leurs personnages : Pamela, Iphigénie. Or si un personnage de tragédie comme Iphigénie peut dire une vérité qui touche à l'essence d'un peuple, c'est donc qu'il y a une vérité du théâtre (comme du roman). Dans un ouvrage qui condamne le théâtre comme un art de la fausseté et de l'artifice, il y a là pour le moins une contradiction. Les ajouts tardifs de Rousseau témoignent d'une position moins ferme à l'égard du message principal de son texte, comme s'il l'ouvrait à une forme de débat, comme si d'une certaine manière l'auteur avait pu se tromper.

Tournons la page. Juste après le développement sur le théâtre à Athènes suit - inévitablement chez Rousseau qui les oppose toujours - un passage sur Sparte 42 : Athènes a pu se laisser séduire par le théâtre, mais Sparte la vertueuse, son modèle politique par excellence, non. Elle a résisté à ses attraits et à ses pièges. Rousseau écrit :

Avec tout cela, jamais la Grece excepté Sparte, ne fut citée en exemple de bonnes mœurs; et Sparte, qui ne soufroit point de Théatre, n'avoit garde d'honorer ceux qui s'y montrent.

Sur l'exemplaire retouché (fig. 3), un astérisque ressemblant presque à une tache d'encre qui aurait éclaboussé la page entraîne l'œil vers le bas : "Voyez sur cette erreur la Lettre de M. Le Roy. » Dans un lieu si stratégique de son exposé, Rousseau a commis une « erreur », c'est son mot. Un lecteur érudit s'en est aperçu très vite et s'est pressé de lui écrire pour qu'il puisse rectifier. Fin octobre 1758, soit quelques semaines à peine après la parution du livre à Paris (le 2 octobre), Rousseau reçoit une lettre de Julien David Le Roy, connaisseur en architecture antique, futur membre de l'Académie des inscriptions et belles-lettres, lui expliquant respectueusement que... non seulement il y avoit un théatre à Sparte, absolument semblable à celui de Bachus à Athenes, mais il étoit le plus bel ornement de cette ville si celebre par le courage de ses habitans, il subsiste même encore en grande partie, et Pausanias et Plutarque en parlent. C'est d'après ce que ces deux auteurs anciens en disent que j'en ai fait L'histoire, que je vous envoie, dans L'ouvrage que je viens de mettre au jour. Comme cette erreur qui vous est échapée, pouroit être remarqué par d'autres que par moi, j'ai cru que vous ne seriez pas faché que je vous en avertisse, et je me flate, Monsieur que vous voudrez bien recevoir ce foible avis, comme une marque de L'estime et de la parfaite consideration avec laquelle j'ai L'honneur d'être votre tres humble et tres Obeissant serviteur. Le Roy 43 .

Rousseau lui répond quelques jours plus tard en s'excusant pour sa «bévue » et en lui exprimant sa reconnaissance : «Il s'en faut peu que je ne me félicite d'une erreur qui m'attire de vôtre part cette marque d'estime, et je me sens moins honteux de ma faute que fier de vôtre correction. » Ce n'est pas qu'il ait manqué de consulter les bons auteurs, mais la faute est là et il ne veut pas avoir la vanité de la méconnaître :

loin de Souhaitter que cette faute reste cachée à mes Lecteurs, je serai fort aise qu'on le publie et qu'ils en Soient instruits. Ce sera toujours une erreur de moins. D'ailleurs, comme elle ne fait tort qu'à moi seul, et que mon Sentiment n'en est pas moins établi, j'espère qu'elle pourra Servir d'amusement aux Critiques ; j'aime mieux qu'ils triomphent de mon ignorance que de mes maximes, et je serai toujo[urs] très content que les vérités utiles que j'ai sou[t] enue[s] [soient] épargnées à mes dépends. Recevez, Monsieur, les assurances de ma reconnoissance, de mon estime, et de mon respect ${ }^{44}$.

40. P. 142. Ce passage correspond à la page 71 de l'édition de la Pléiade. Cette note, qui a bien été ajoutée dans l'édition de 1781, a échappé aux éditeurs de la Pléiade dans leur collationnement.

41. Euripide, Iphigénie en Aulide. Rousseau a probablement à l'esprit la traduction du père Brumoy parue dans son Théâtre des Grecs, 1730. Nous la citons d'après une réédition : Théâtre des Grecs, par le P. Brumoy, nouvelle édition, Paris, Cussac, 1786, t. VII, p. 236.

42. Ce passage correspond à la page 72 dans l'édition de la Pléiade.

43. Lettre de Julien David Le Roy à Rousseau, 29 octobre 1758, CC, lettre $\mathrm{n}^{\circ} 721$.

44. Lettre à J. D. Le Roy, 4 novembre $1758, C C$, lettre $n^{\circ} 725$. 
Au lieu d'écrire à son éditeur, de lui demander sur le champ des cartons rectificateurs à placer dans les éditions que celui-ci est en train de diffuser, ce qui dissimulerait sa faute et le sauverait d'un certain ridicule, Rousseau choisit une tout autre voie : exhiber sa bévue ainsi que sa rectification grâce à autre que lui. Que signifie l'ajout autographe successif, tracé avec une plume taillée plus finement et d'une encre différente : « Dans le Tome sixième »? Cette mention se comprend en référence au projet d'édition générale de ses écrits que Rousseau prépare et qu'il communique notamment à Rey en 1764. Selon les vœux de Rousseau, cette édition devra comporter un sixième et dernier tome qui « contiendra les piéces de theatre, ouvrages de litterature, lettres et mémoires 45 », et dans lequel le lecteur pourra trouver la lettre de Le Roy... Tout cela ne sera pas réalisé par Rey ni par d'autres éditeurs alors en concurrence, mais par les éditeurs de 1781 qu'on voit à l'œuvre sur la page, hésitant et débattant entre eux pour savoir comment intégrer ce renvoi et trouvant, après plusieurs ratures - « NB. Ce renvoi est embarrassant, ta Lettre eitée à $M$. le Roy ne m'étant jusqu'à présent point eonnuie. Si elle se trouve dans les papiers de l'Auteur, il faudra indiquer ee renvoi au Tome où elle sera insérée. »- la bonne solution : «On la trouvera dans la collection des Lettres de M. Rousseau, à la fin de ce recueil ».

Pourquoi Rousseau n'a-t-il pas modifié son passage et a-t-il voulu mettre sous les yeux des lecteurs sa propre « erreur »? Avant de répondre, avançons encore trois pages plus loin. Rousseau a encore durci le ton contre les comédiens et continue sa diatribe. À force de jouer le rôle de valets filous, ne deviendront-ils pas des fripons et des voleurs à leur tour : «Ne prendront-ils jamais la bourse d'un fils prodigue ou d'un pere avare pour celle de Léandre ou d'Argan ? ( (p. 145, fig. 13). De nouveau, une note infrapaginale manuscrite arrête la lecture :

On a relevé ceci comme outré et comme ridicule. On a eu raison. Il n'y a point de vice dont les comédiens soient moins accusés que de la friponnerie. Leur métier qui les occupe beaucoup et leur donne même des sentimens d'honneur à certains égards, les éloigne d'une telle bassesse. Je laisse ce passage, parce que je me suis fait une loi de ne rien ôter ; mais je le desavoue hautement comme une très grande injustice.
C'est un spectaculaire reniement de la part de Rousseau : on a eu raison, j'ai eu tort et je le dis « hautement 46 . Si Rousseau n'a plus son Aristarque judicieux et sévère pour passer au crible ce qu'il écrit, il en a bien d'autres et au premier chef lui-même, qui est à son propre égard particulièrement sévère. De nouveau, au lieu de dissimuler une erreur, Rousseau la laisse sous les yeux de ses lecteurs. Il la met même en lumière par un désaveu assez théâtral. À travers la rectification mise en scène de deux erreurs successives, ces notes manuscrites ajoutées dans les marges de la Lettre à D'Alembert posent la question du vrai et du faux, racontent la manière dont la vérité d'une parole se construit, se déconstruit, peut être rectifiée individuellement ou collectivement, et doit même l'être quoi qu'il en coûte. Car Rousseau n'y était-il pas obligé par ses propres principes ? Ne s'était-il pas choisi pour devise en 1758, dans une note en bas de la Lettre à D'Alembert justement, le vitam impendere vero (consacrer sa vie au vrai) de Juvénal47 ? En se relisant, en se révisant, il allait mettre en pratique un tel engagement. Reste un problème : à sa mort, ces notes étaient encore inédites. Son souci de la vérité fut couché sur le papier, mais remis à plus tard, à la postérité.

L'exemplaire retouché de la Lettre à D'Alembert nous fait découvrir une œuvre vivante et en mouvement au-delà de l'impression qui pouvait la fixer dans sa forme de 1758. La démarche adoptée dans cette étude a cherché à considérer ces retouches de Rousseau dans leur organicité, et à considérer ce travail long de révision comme un acte cohérent et interprétable. D'après un certain nombre d'indices, il semble que Rousseau ait mené ce travail entre l'automne 1758 et l'année 1764. Un dialogue prolongé se tisse alors entre Rousseau, son œuvre, ses lecteurs, ses propres lectures, à l'issue

45. Lettre à Rey du 13 mai 1764, CC, lettre n 3273.

46. Ce « on » dont Rousseau tait le nom est le Dancourt de Berlin dont nous avons parlé précédemment. Il critique précisément ce passage à la page 274 de sa Lettre à J.-J. Rousseau.

47. Devise que Dancourt n'avait pas manqué de parodier dans sa réponse à la Lettre à D'Alembert en lui opposant le «ridendo dicere verum » d'Horace, placé en épigraphe de son pamphlet. 
duquel la force polémique de la Lettre à D'Alembert, machine de guerre qui était tournée d'abord toute vers ses adversaires, s'est en quelque sorte intériorisée. Au nom de la vérité, quelques notes infrapaginales contestent l'auteur même et font violence à sa parole. Est-ce le signe d'un orgueil sans bornes, d'une suprême maîtrise, ou bien l'indice de la fêlure qui conduira aux Dialogues? À d'autres de le dire.

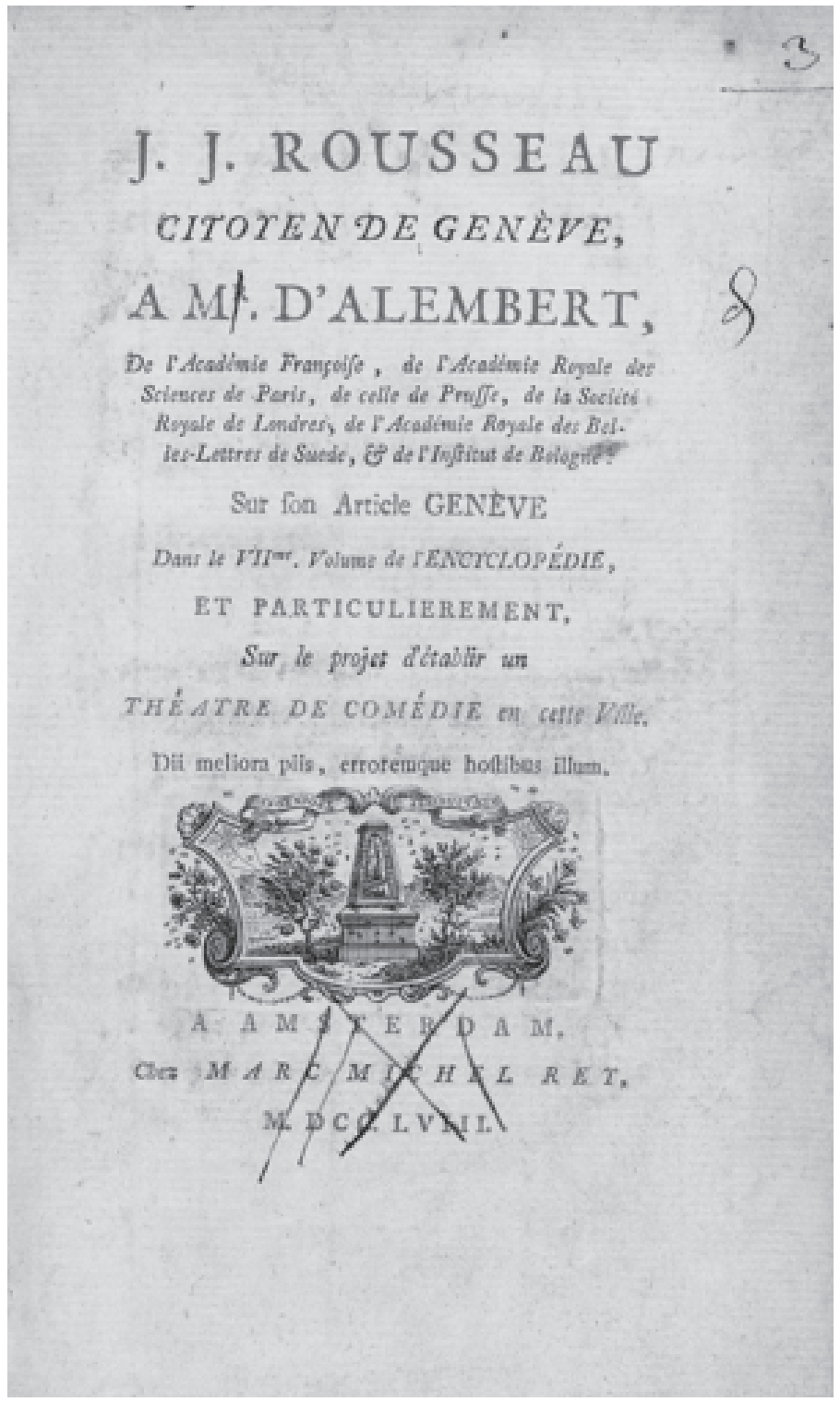




\section{J. J. ROUSSEA U \\ CITOTEN DE GENEVE,}

\section{A Monsieur D'Alembert.}

25.

2 J 30 re article, GENEVE, dans

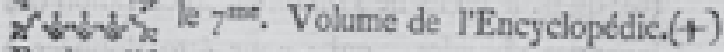

En le relifant avee plas de plaifir encore, il m'a fourni quelques réflexions que j’ai crù pouvoir offrir, fous vos aufpices, au public \& à mes Concitoyens. Il y a besucoup à louer dans cet article; mais fi les eloges dont vous honoret. ma Patrie m'ôtent le droit de vous en rendre, ma fincérité parlera pour moi ; n'ětre pas dé votre avis fur quelques points, c'eft affic: m'expliquer fur les aatres.

$\mathrm{J}$ E commencerai par celui que jai le plus de répugnance à traiter, \& done rexamen me convient te moins; mais fur lequel, par h raifon qque je viens de dire, $k$ filence ne m'eft pas permis. Ceft le jugement que wous portez de ha doctrine de nos. Miniftres en matiere de foi.

\section{A}

Vous

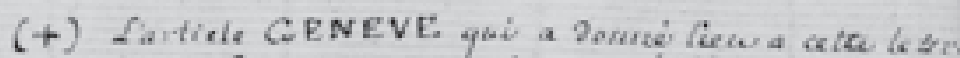

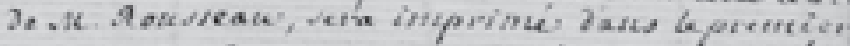

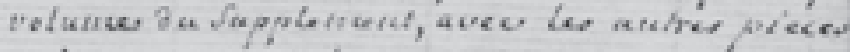

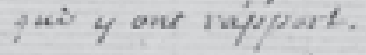

\section{Fig. 2}

(+) L'article GENEVE qui a donné lieu à cette lettre de M. Rousseau, sera imprimé dans le premier volume du Supplement, avec les autres pièces qui y ont rapport. 


\section{J. J. ROUSSEAU}

n'offroient de toutes parts que des combats, des vietoires, des prix, des objets capables d'infpirer aux Grecs une ardente émulation, \& d'échauffer leurs caurs de fenthimens d'honneur $\&$ de gloire. C'eft au milieu de cet impofant appareil , fi propre à elewcr \& remuer l'ame, que les Aeteurs, animis da méme zele, partageoient, fclon' leurs talens, les honneurs rendus aux vainqueurs des jeux, fouvent atx premiers hommes de la nation. Je ne fuis pas furpris que, loin de les avilir, leur métier, cxercé de cette maniere, leur donnat sette ficrté de courage \& ce noble défintéreflement qui fembloit quelquefois elever PActeur à fon perfonnage. Avee tout cela, jamais la Grece, exccpté Sparte, ne fut citée en exemple de bonnes mours; \& Spar. te, qui ne fouffroit point de Théatre"; n'avoit garde d'honorer ceux qui s'y montrent.

Revenoss aux Romains qui, loin de fuivre à cet egard lexemple des Gress, en donnerent un tout contraire. Quand leurs bix déelaroient les Comediens infumes, étoit-

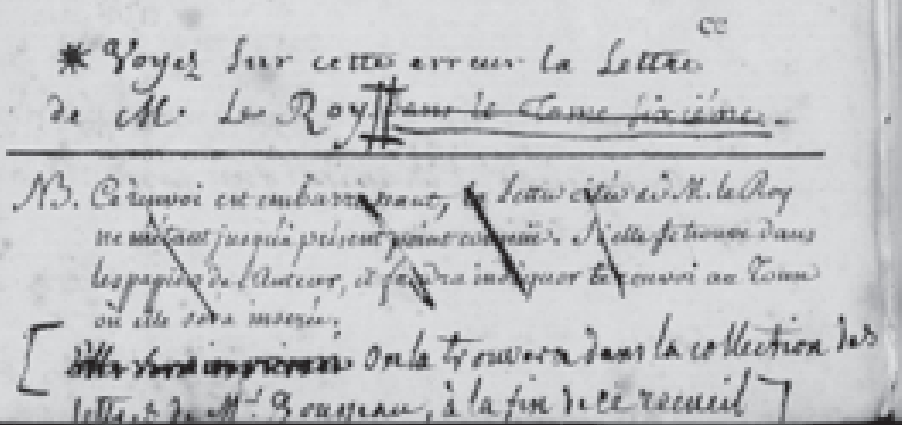

Fig. 3

* Voyez sur cette erreur la Lettre de M. Le Roy. Đans le Tome Sixième.

NB. Ce renvoi est embarrassant, la Lettre citée à M. le Roy ne m'étant jusqu'à présent point eonnüe. Si elle se trouve dans les papiers de l'Auteur, il faudra indiquer ee renvoi au Tome où elle sera insérée.

[Elle sera imprimée On la trouvera dans la collection des Lettres de Mr Rousseau, à la fin de ce recueil] 
A Mr. D'ALEMBERT.

perfuade que, pour être honnétc-homme, il fuffit de n'étre pas un franc feclérat.

J'A R $\mathrm{R}$ 1s trop d'avantage, fi je voulois paffer de lexamen de Moliere à celui de fes fuceeffeurs, qui, n’ayant ni fon génie, ni fa probité, n'cn ont que mieux fuivi fes voes intéreflées, en s'attachant à flatter une jeuneffe debanchíe \& des femmes fans moeurs. I Mne ferai pas à Dancourt l'honneur parler dovi : fes Pieces n'effaroachent pas par des termo obfenes, mais nat navoir de chaite que les oncilles/pour les pouvoir fupporter. Regnasu, Plus modefte, n'eft pas moins dapgereux : laiflant Kutre amufer les ferpars perdues, il fe charge, lin, diencen-

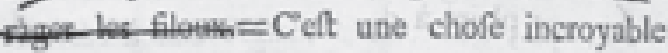
quavec l'agrément de la Police, on joue publiquement au milieu de Paris une Comédie, où, dans Tappartement d'ua oncle qu'on vient de voir expirer, fon neveu, l'honnetehomme de la Piece, s'occupe avee fon digne cortege, de foins que les loix paicnt de la corde; \& qu'au lieu des larmes que la feale humanité fait verfer en pareil cas aux indiffe-

$$
\text { E } 5
$$

rens
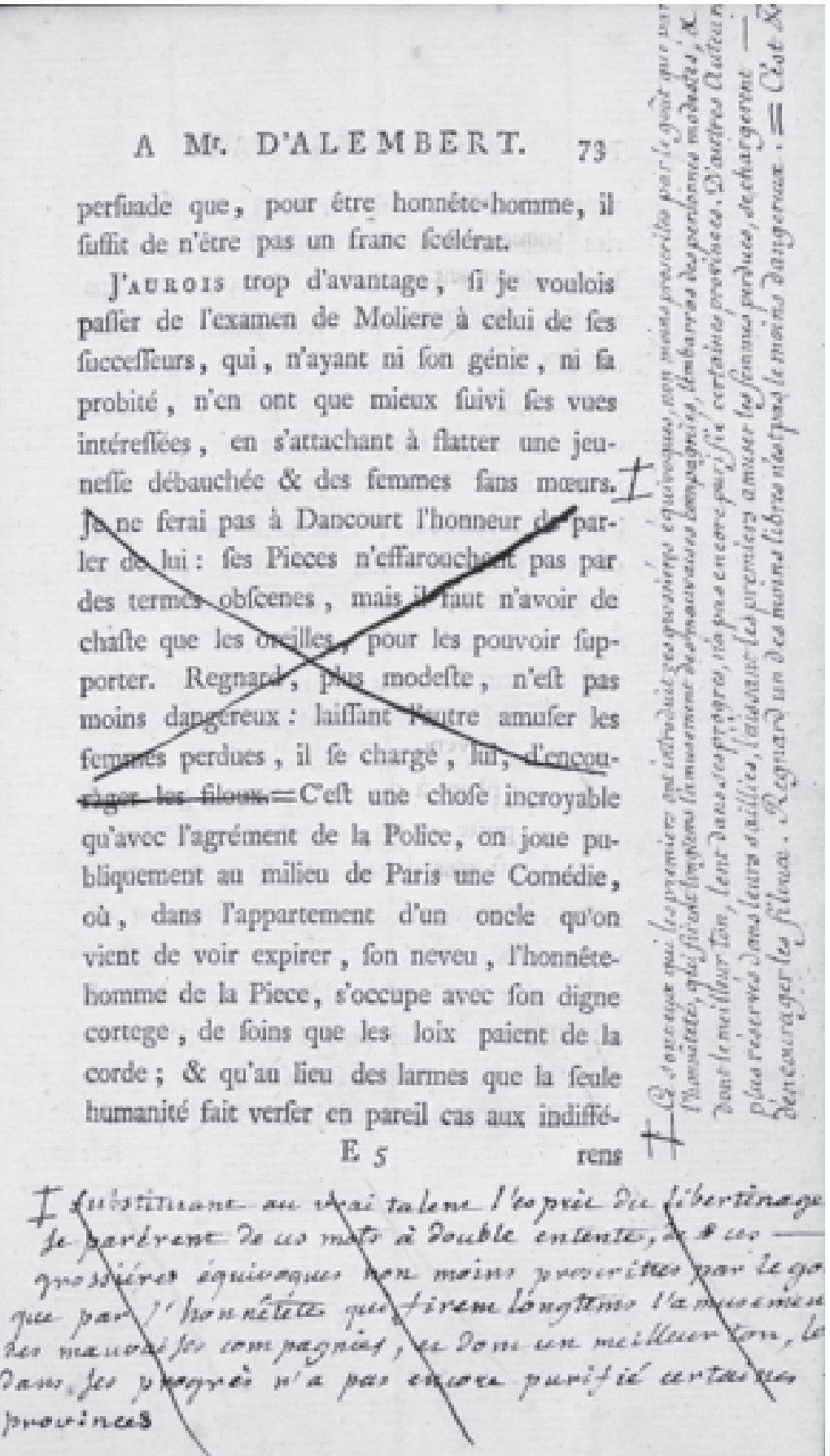

Fig. 4

substituant au vra talent l'esprit du libertinałe se parérent de ces mots à double entente, de 1 ces grpssiéres équivoques non mbins proscrittes par le goût que par l'honnêteté qui firent longtems l'amusement des mauvaises compagnies, et dor t un meilleur ton, lent dans es progrès n'a pas encore purifié certaines provinces

[marge droite] Ce sont eux qui les premiers ont introduit ces grossiéres équivoques, non moins proscrites par le goût que par l'honnêteté, qui firent longtems l'amusement des mauvaises compagnies, l'embarras des personnes modestes, \& dont le meilleur ton, lent dans ses progres, n'a pas encore purifié certaines provinces. D'autres Auteurs, plus réservés dans leurs saillies, laissant les premiers amuser les femmes perdues, se chargerent d'encourager les filoux. Regnard un des moins libres n'est pas le moins dangereux. $=$ C'est $\& \mathrm{c}$ 
A Mr. D'ALEMBERT. 57

fon ami trahir lachement fon fentiment \& tromper I'bomme qui le lui demande, il s'en voit encore plaifanter lui - méme au plus fort de fa colere. Il eft naturel que cette colere dégenere en emportement \& lui fafle dire alors plus qu'il ne penfe de fang-froid. D'ailleurs, la raifon qu'il rend de cette haine univerfelle en juftifie pleinement la caufe.

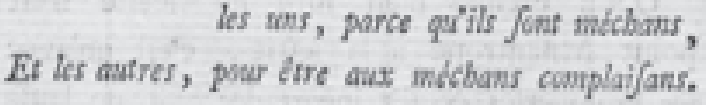
Ce n'eft done pas des hommes qu'il cft cnnemi, mais de la méchanceté des uns \& da fupport que cette méchanceté trouve dans les autres. S'il n'y avoit ni frippons, ni flatteurs, il aimeroit tout le gemonde hat no n'y a pas un homme de bien qui ne foit Mifantrope en ce

fens;

re, \& n'ayant pour tous matériaux qu'un confus fouvenir des obfervations que jai faites autrefois au Spectacle, je puis me tromper dans mes cita. tions \& renverfer Pordre des Pieces. Mais quand mes exemples feroient peu juftes, mes raifons ne le-feroient pas molns, attendu qu'elles ne font point tirées de telle ou telle Piece, mais de lef. ptit gétactral da Théatre, que jai bien éradié.

$$
\text { D } 5
$$

Fig. 5 
A M. D'ALEMBERT. 55

joucr celui que lé monde pardonne le mains, le nidicule de la vertu: c'eft ce qu'il a fait dans le Mifantrope.

Vous ne furiez me nicr deux chofes: lune, qu'Alcefle dans cette Piece eft un homme droit, fincere, eftimable, un véritable homme de bicn; Tautre, que l'Auteur lui donne un perfonnage ridicule. C"en eft affè's, ce me femble, pour rendre Moliere inexcufable. On pourroit dire qu'il_a joué dans Al. cefte, non la vertu, mais un véritable défaut, qui eft $\mathrm{A}$ haine des hommes. "A cela je ré. ponds qu'il n'eft pas vrai quilil ait donné cette haine a fon perfonnage: il ne faut pas que ce nom de Mifantrope en impofe, comme fi ce. lui qui le porte etoit ennemi du genre humain. Une purcille haine ne feroit pas un defaut, mais une dépravation de la Nature \& le plus grand de tous les vices: puifyue, touter tes

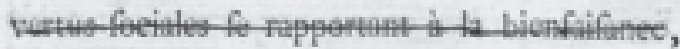

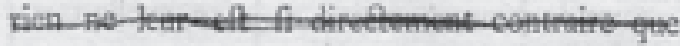

Lindmantite. Le vrai Mifantrope eft un monftre. S'il pouvoit exifter, il ne feroit pas rite; il feroit horreur. Vous pouvez avoir vu $\mathrm{D}_{4}$

Fig. 6 
ftance \& le fujet le comportent: n'eft-il pas clair, par exemple, qu'a moins qu'un Auteur ne foit de la derniere mal-adreffe, un tel difcours doit faire fondre en larmes toute l'affemblee? La Piece, finiflant ainfi, fira, fi roa veut, moins bonne, moins inftructive, moins conforme a l'hiftoire, mais en fera-telle moins de plaifir, \& les Speetateurs en fortiront-ils moins fatisfaits ? Les quatre premiers Actes fubfifteroient à peu prés tels guils font, \& eependant on cn tircroit une legoon directement contraire. Tant il eft vrai que les tableaux de Pamour font toujours plus d'impreffion que les maximes de la fageffe, \& que l'cffet d'une Tragédie eft tout-àfait indépendant de celui da dénouement! ${ }^{\mathbf{k}}$

$\mathrm{V}$ guT-oN favoir s'il eft fur qu'en montrant les fuites funeltes des paffions immodé. rées, la Tragédie apprenne à s'en garantir? Que l'on confulte l'expérience. Ces fuites funcftes font repréftntées très fortement dans Zuire; il en coute la vie aux deux Amans, $\&$ il en conte bien plas que la vie à Orofmane: puiffu'il ne fe donne la mort que pour

\section{fo}

* H y a zans le $7^{e}$ bome de Damela, un expmun

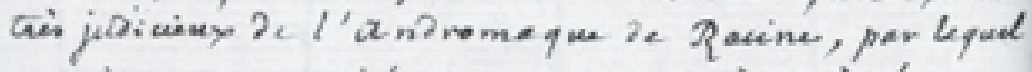

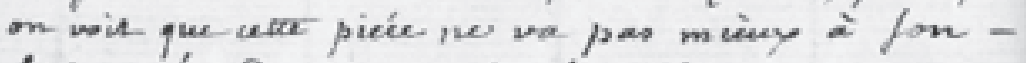

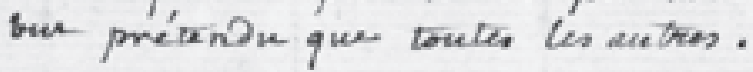

Fig. 7

* Il y a, dans le 7e tome de Pamela, un examen très judicieux de l'Andromaque de Racine, par lequel on voit que cette piéce ne va pas mieux à son but prétendu que toutes les autres. 
A Mr. D'ALEMBERT. 95

jamais régner fur la Scene qu'entre des ames honnetes, c'eft que les deux Amans font toujours des modeles de perfection. Et comment ne s'intérefferoit-on pas pour une paffion fi féduifante, entre deux courrs dont le caractere eft déja fi intéreffant par lai-mème? Je doute que, dans toutes nos Pieces dramatiques, on en trouve une feule où l'amour mutuel n'ait pas la faveur du Spectateur. Si quelque infortuné brâle d'un feu non partagé, on en fait le rebut du Parterre. On croit faire merveilles de rendre un amant eftimable ou haiffable, felon qu'il eft bien ou mal accueilli dans fes amours; de faire toujours approuver au public les fentimens de fa maitreffe; \& de donner à la tendrefle tout l'intérêt de la vertu. Au-licu qu'il faudroit apprendre aux jeunes'-gens à fe défier des illufions de l'amour, à fuir Perreur d'un penchant aveugle qui croit toujours fe fonder fur leftime, \& à craindre quelquefois de livrer un caur vertueux à un objet indigne de fes foins. Je ne fache gueres que le Mifuntrope où le héros de la Piece ait fait un mavais choix.*

Ren.

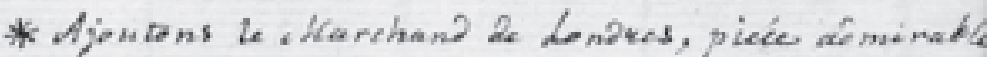

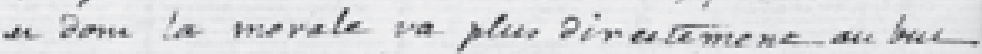

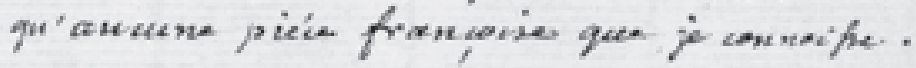

Fig. 8

* Ajoutons le Marchand de Londres, piéce admirable et dont la morale va plus directement au but qu'aucune piéce françoise que je connoisse. 
A Mr. D'A LEMBERT. 14I

toire de leur pays. $4^{\circ}$. Ce Peuple, enthou. fiafte de fa liberté jufquà croire que les Grees étoicnt les feuls hommes libres par nature, fe rappelloit avec un vif fentiment de plailir fes anciens malheurs \& les crimes de fes Maitres. Ces grands tableaux linftruifoient fans ceffe, \& il ne pouvoit fe défendre d'un peu de refpet pour les organes de cette inftruetion. $5^{\circ}$. La Tragédie n'étant d'abord jouće que par des hommes, on ne voyoit point, fur leur Théatre, ce mélange feandaleux d'hommes \& de femmes qui fait des nồcres autant d'écoles de mauvaifes maurs. 6o. Enfin leurs Speetacles n'avoient rien de la mefquinerie de ceux d'aujourd'hai. Leurs Théatres n'étoient point élevés par lintérét \& par l'avarice ; ils n'étoient point renfelthés dans d'obfcures prifons; leurs Acteurs n'avoient pas befoin de mettre à contribution les Spectateurs, ni de compter du coin de l'ail les gens qu'ils voyoient paffer la porte, pour être furs de leur fouper.

CEs grands \& fuperbes Speetacles donnćs fous le Ciel, à la face de toute une nation, n'of-

* Iphiqúnie los die en rermes eppies Dan

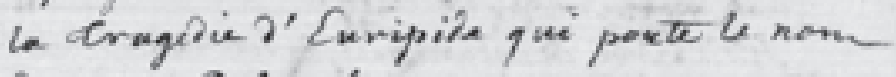

De cute Drinapo.

Fig. 9

* Iphigénie le dit en termes exprès dans la Tragedie d'Euripide qui porte le nom de cette Princesse. 


\section{J. J. ROUSSEAU}

les Comédiens de Geneve fe foutiendront avee une affemblée de quarante huit Specta. teurs pour toute reffource? Vous me direz qưon vit à meilleur compte à Geneve quä Paris. Oui, mais les billets d'entrée coutteront auffi moins à proportion; \& puis, la dépenfe de la table n'eft rien pour des Comédiens. Ce font les habits, c'eft la parure qui leur côte; il fandra faire venir tout cela de Paris, ou dreffer des Otuviers mal adroits. "C'eft' dans les lieux où toutes ces chofes font communes qu'on les fait à meilleur marché. Vous direz encore qu'on les affijjétira à nos loix fomptuaires. Mais c'eft en vain qu'on voudroit porter la réforme fur le Théatre; jamais Cléopatre \& Xercès ne goateront no. tre fimplicité. L'état des Comédiens étant de paroître, c'eft leur ôter le goût de leur métier de les en empêcher, \& je doute que ja. mais bon Acteur confente à fe faire Quakre. Enfin, lon peut m'objetter que la Troupe

bons \& mauvais jours, la troaveront farement trop

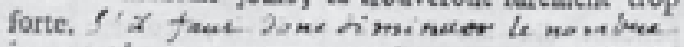

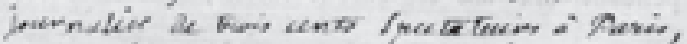

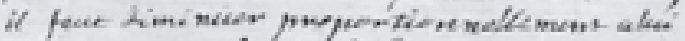

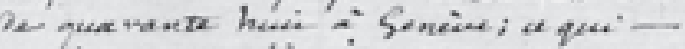
renforce mes blictions.

Fig. 10

S'il faut donc diminuer le nombre journalier de trois cents spectateurs à Paris, il faut diminuer proportionnellement celui de quarante huit à Genève ; ce qui renforce mes objections. 
hommes, les femmes nous rendent femmes.

$\mathrm{C}_{\boldsymbol{E}} \mathrm{T}$ inconvénicnt qui dégrade l'homme, eft très grand par-tout ; mais c'eft fur-tout dans les Etats comme le nôtre qưiil importe de le prévenir. Qu'un Monarque gouverne des hommes ou des femmes, eela lui doit étre affés indifférent pourvu qu'il foit obéi; mais dans une République, il faut des hom. mes ( $g$ ).

L s s Anciens paffoient prefque leur vie en plein air, ou vacquant à leurs affaires, ou réglant eelles de I'Etat fur la place publique, oa fe promenant à la campagne, dans des jardins, an bord de la mer, à la pluie, au

foleil,

(g) On me dira qu'il en faut aux Rois pour la guerre. Polnt du tout. Au-lieu de trente mille hommes, ils n'oot, par exemple, ga'a lever cent mille femmes. Les femmes ne manquent pas de courage: elles préferent I'honneur ì la vie; quand elles fe bartent, elles fe battent bien. L'incos. vénient de leur fexe efl de ne pouvoir fupporter les fatigues de la guerre \& l'intempérie des fai. fons. Le focret eft done-d'en avoir toujours le trip̧le de ce qu'il en faot pour, fe battre, afin do facrifer les deux autres tiers aux maladies o a la mortalité.

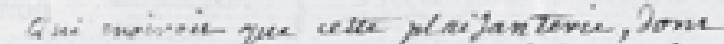

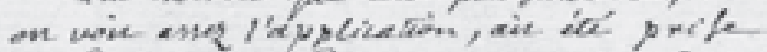

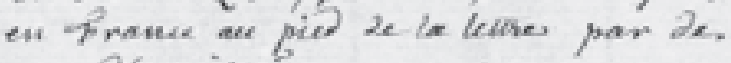
gens oropict?

Fig. 11

Qui croiroit que cette plaisanterie, dont on voit assez l'application, ait été prise en France au pied de la lettre par des gens d'esprit? 
A Mr. D'ALEMBERT, 20i

ment corrompues ne fauroient fupporter long. tems cette maniere de vivre, \& quclque che. re que leur pût être la médifance, elles vou. droient médire avee des hommes. Quoiqu'on m'ait pa dire à cet égard, je n'ai jamais vo aucune de ces fociétés, fans un fecret mouvement d'eftime \& de refpest pour celles qui $\mathrm{l}_{\mathrm{a}}$ compofoient. Telle eft, me difois-je, la deftination de la Nature, qui donne différens golts aux deux fexes, afin qu'ils vivent fépa. rés \& chacun à fa maniere (1). Ces aimables perfonnes paffent ainfi leurs jours, livrées aux occupations qui leur conviennent, ou à des amufemens innocens \& fimples, très pro. pres à toucher un cour honnête \& à donner bonne opinion d'elles. Je ne fais ee qu'elles ont dit, mais elles ont vicu enfem. ble; elles ont pu parler des bommes, mais

(1) Ce principe, auquel tiennent toutes bonnes mexurs, eft dfveloppe dane maniere plus chaire \& plas étendue dans un manufcrit dont je fais dépofitnire a que je me propole de publier, s'it me rufte, affés de tems pour celn, quooignue cette annonce $\mathrm{se}$ foit gueres propre il lal concilier davance la faveur des Dames.

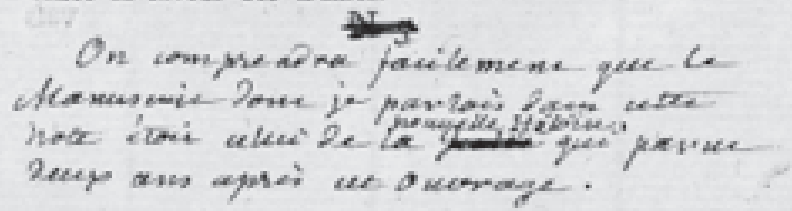

Fig. 12

On comprendra facilement que le Manuscrit dont je parlais dans cette nouvelle Héloïse

note étoit celui de la Julie qui parut deux ans après cet ouvrage. 


\section{A Mr. D'ALEMBERT. 145}

pour la perfonne quilil repréfente, ni qu'on le crole affocté des paffions qu'il imite, \& qu'cn donnant ectte imitation pour ce qu'elle cft, il la rend tout à fait innocente. Auffi ne laccufé.je pas d'être précifiment un trompeur, mais de eultiver pour tout métier le talent de tromper les hommes, \& de s'exercer à des habitudes qui, ne pouvant être innocentes qu'au Theatre, ne fervent par - tout ailleurs gu'à mal faire. Ces hommes fi bien parćs, fi bien exerés au ton de la galanterie $\&$ aux accens de la paftion, n'abuferontils jamais de ect art pour féduire de jeanes perfonnes? Ces valets filour, fi fubtils de la langue \& de Ja main far la Seene, dans les befoins d'un métier plas difpendieux que lucratif, n'auront-ils jamais de diftractions utiles? Ne prendront-ils jumais la bourfe d'un fils prodigue ou d'un pere avare pour celle de Léandre ou d'Argant? Par-tout la tentation de mal faire angmente avec la facilité; \& il faut que les Comb́diens foient plus vertueux que les autres hommes, sils ne font pas plus corrompas.

K

L'O.

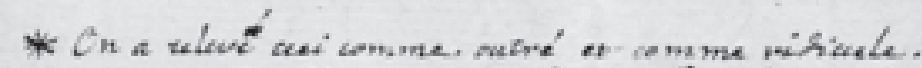

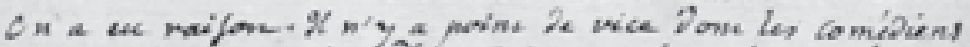

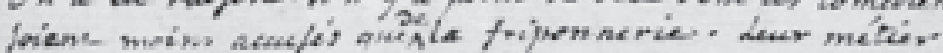

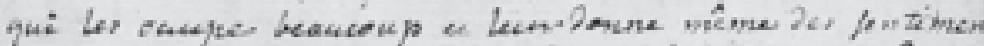

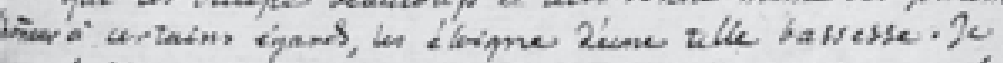

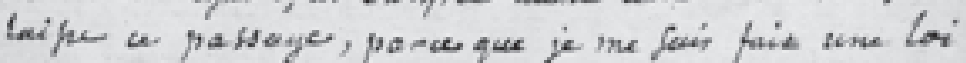

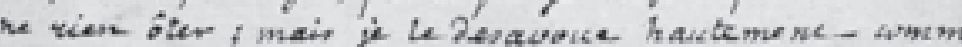
ture tais arane in justice.

Fig. 13

* On a relevé ceci comme outré et comme ridicule. On a eu raison. Il n’y a point de vice dont les comédiens soient moins accusés que de la friponnerie. Leur métier qui les occupe beaucoup et leur donne même des sentimens d'honneur à certains égards, les éloigne d'une telle bassesse. Je laisse ce passage, parce que je me suis fait une loi de ne rien ôter ; mais je le desavoue hautement comme une très grande injustice. 